\title{
Weighted Trudinger inequality associated with rough multilinear fractional type operators
}

Han Feng and Qingying Xue*

"Correspondence: qyxue@bnu.edu.cn Laboratory of Mathematics and Complex Systems, School of Mathematical Sciences, Beijing Normal University, Ministry of Education, Beijing, 100875, People's Republic of China

\begin{abstract}
Let $\Theta_{\Omega, \alpha}^{\Theta}$ be the multilinear fractional type operator defined by

$\Theta_{\Omega, \alpha}^{\Theta}(\vec{f})(x)=\int_{\mathbb{R}^{n}} \Omega(y) \prod_{j=1}^{m} f_{j}\left(x-\theta_{j} y\right)|y|^{(\alpha-n)} d y$. In this paper, we study the weighted estimates for the Trudinger inequality associated to $I_{\Omega, \alpha}^{\Theta}$ with rough homogeneous kernels, which improve some known results significantly. A similar Trudinger inequality holds for another type of fractional integral defined by $\bar{I}_{\Omega, \alpha}(\vec{f})(x)=\int_{\left(\mathbb{R}^{n}\right)^{m}} \frac{\prod_{j=1}^{m}\left|f_{j}\left(y_{j}\right)\right| \Omega_{j}\left(x-y_{j}\right) \mid}{\mid\left(x-y_{1}, x-y_{2}, \ldots, x-y_{m}\right)^{m n-\alpha}} d \vec{y}$, where $d \vec{y}=d y_{1} \cdots d y_{m}$.
\end{abstract}

Keywords: Riesz potential; multilinear fractional integral; $A_{p}$ weights; $A_{p, q}$ weights; Trudinger inequality

\section{Introduction}

The Trudinger inequality (also sometimes called the Moser-Trudinger inequality) is named after N. Trudinger who first put forward this inequality in [22]. Later, J. Moser [14] gave a sharp form of this Trudinger inequality. It provides an inequality between a certain Sobolev space norm and an Orlicz space norm of a function. In [14], J. Moser gave the largest positive number $\beta_{0}$, such that if $u \in C^{1}\left(\mathbb{R}^{n}\right)$, normalized and supported in a domain $D$ with finite measure in $\mathbb{R}^{n}$, such that $\int_{D}|\nabla u(x)|^{n} d x \leq 1$, then there is a constant $c_{0}$ depending only on $n$ such that for all $\beta \leq \beta_{0}=n w_{n-1}^{1 /(n-1)}$, where $w_{n-1}$ is the area of the surface of the unit $n$-ball. The following inequality holds:

$$
\int_{D} \exp \left(\beta|u(x)|^{n /(n-1)}\right) d x \leq c_{0}|D| .
$$

In 1971, D. Adams [1] considered the similar inequality of J. Moser for higher order derivatives. The key, for him, was to write the function $u$ as a potential $I_{\alpha}$ (see the definition below) and prove the analogue of (1.1) as follows:

$$
\int_{D} \exp \left(\frac{n}{w_{n-1}}\left|\frac{I_{\alpha} f(x)}{\|f\|_{p}}\right|^{n /(n-\alpha)}\right) d x \leq c_{0}|D|, \quad \text { for } \alpha=n / p, f \in L^{p}(1<p<\infty) .
$$

Variant forms of the Trudinger inequality as a generalization of the classical results, especially in the literature associated with multilinear Riesz potential or multilinear fractional integral, have been studied in recently years (see, for example, $[2,3,6,7,10,14,16-$ 18 ,

๑ 2012 Feng and Xue; licensee Springer. This is an Open Access article distributed under the terms of the Creative Commons Attribution License (http://creativecommons.org/licenses/by/2.0), which permits unrestricted use, distribution, and reproduction in any medium, provided the original work is properly cited. 
20, 21]). This kind of inequality plays an important role in Harmonic analysis and other fields, such as PDE.

We begin by introducing a class of multilinear maximal function and multilinear fractional integral operators. Suppose that $n \geq 2,0<\alpha<n, \Omega$ is homogeneous of degree zero, and $\Omega \in L^{s}\left(S^{n-1}\right)(s>1)$, where $S^{n-1}$ denotes the unit sphere of $\mathbb{R}^{n}$. The multilinear maximal function and multilinear fractional integral is defined by

$$
I_{\Omega, \alpha}^{\Theta}(\vec{f})(x)=\int_{\mathbb{R}^{n}} \Omega(y) \prod_{j=1}^{m} f_{j}\left(x-\theta_{j} y\right)|y|^{(\alpha-n)} d y
$$

and the fractional maximal operator $M_{\Omega, \alpha}$ defined by

$$
M_{\Omega, \alpha}^{\Theta}(\vec{f})(x)=\sup _{r>0} \frac{1}{r^{n-\alpha}} \int_{|y|<r}|\Omega(y)| \prod_{j=1}^{m}\left|f_{j}\left(x-\theta_{j} y\right)\right| d y .
$$

Multilinear fractional integral $I_{\Omega, \alpha}^{\Theta}$ can be looked at as a natural generalization of the classical fractional integral, which has a very profound background of partial differential equations and is a very important operator in Harmonic analysis. In fact, if we take $K=1$, $\theta_{j}=1$, and $\Omega=1$, then $I_{\Omega, \alpha}^{\Theta}$ is just the well-known classical fractional integral operator studied by Muckenhoupt and Wheeden in [15]. We denote it by $I_{\alpha}$. If $\Omega \equiv 1$, we simply denote $I_{\Omega, \alpha}^{\Theta}=I_{\alpha}^{\Theta}$. In recent years, the study of the Trudinger inequality associated to multilinear type operators has received increasing attention. Among them, it is well known that Grafakos considered the boundedness of a family of related fractional integrals in [7]. After that, in [6], Y. Ding and S. Lu gave the following Trudinger inequality with rough kernels.

Theorem A ([6]) Let $0<\alpha<n, s=\frac{n}{\alpha}, \frac{1}{s}=\frac{1}{p_{1}}+\frac{1}{p_{2}}+\cdots+\frac{1}{p_{m}}, p_{j}>1, j=1,2, \ldots, m, m \geq 2$. Denote $B$ as a ball with a radius $R$ in $\mathbb{R}^{n}$. If $f_{j} \in L^{p_{j}}(B)$, $\operatorname{supp}\left(f_{j}\right) \subset B$, and $\Omega \in L^{n /(n-\alpha)}\left(S^{n-1}\right)$, then for any $\gamma<1$, there is a constant $C$, independent of $n, \alpha, \theta_{j}, \gamma$, such that

$$
\int_{B} \exp \left(n \gamma\left(\frac{L I_{\Omega, \alpha}^{\Theta}(\vec{f})(x)}{\|\Omega\|_{L^{n /(n-\alpha)}} \prod_{j=1}^{m}\left\|f_{j}\right\|_{L^{p_{j}}}}\right)^{n /(n-\alpha)}\right) d x \leq C R^{n},
$$

where $L=\prod_{j=1}^{m}\left|\theta_{j}\right|^{n / p_{j}}, \Theta=\left(\theta_{1}, \theta_{2}, \ldots, \theta_{m}\right), \vec{f}=\left(f_{1}, f_{2}, \ldots, f_{m}\right)$ and

$$
\|\Omega\|_{L^{n /(n-\alpha)}}=\left(\int_{S^{n-1}}|\Omega(x)|^{n /(n-\alpha)} d \sigma(x)\right)^{(n-\alpha) / n} .
$$

The definition of multiple weights $A_{\vec{p}, q}$ was given in [5] and [13] independently, including some weighted estimates for a class of multilinear fractional type operators. These results together with [12] answered an open problem in [8], namely the existence of the multiple weights.

In 2010, W. Li, Q. Xue, and K. Yabuta [16] obtained the weighted estimates for the Trudinger inequality associated to $I_{\alpha}^{\Theta}$ as follows.

Theorem B ([16]) Let $0<\alpha<n, s=\frac{n}{\alpha}, \frac{1}{s}=\frac{1}{p_{1}}+\frac{1}{p_{2}}+\cdots+\frac{1}{p_{m}}, p_{j}>1, \omega_{j}(x) \in A_{p_{j}}$, and $\omega_{j} \geq 1$, $j=1,2, \ldots, m, m \geq 2, v_{\vec{\omega}}=\prod_{j=1}^{m} \omega_{j}^{s / p_{j}}$. Denote $B$ as a ball with the radius $R$ in $\mathbb{R}^{n}$, iff $f_{j} \in L_{\omega_{j}}^{p_{j}}(B)$, 
$\operatorname{supp}\left(f_{j}\right) \subset B, j=1,2, \ldots, m$, then for any $\gamma<1$, there is a constant $C$, independent of $n, \alpha, \theta_{j}$, $\gamma$, such that

$$
\int_{B} \exp \left(\frac{n}{\omega_{n-1}} \gamma\left(\frac{L I_{\alpha}^{\Theta}(\vec{f})(x)}{\prod_{j=1}^{m}\left\|f_{j}\right\|_{L_{\omega_{j}}^{p_{j}}}}\right)^{n /(n-\alpha)}\right) v_{\vec{\omega}} d x \leq C \prod_{j=1}^{m} \omega_{j}(B),
$$

where $L=\prod_{j=1}^{m}\left|\theta_{j}\right|^{n / p_{j}}, \Theta=\left(\theta_{1}, \theta_{2}, \ldots, \theta_{m}\right), \vec{f}=\left(f_{1}, f_{2}, \ldots, f_{m}\right)$.

On the other hand, in 1999, Kenig and Stein [11] considered another more general type of multilinear fractional integral which was defined by

$$
I_{\alpha, A}(\vec{f})(x)=\int_{\left(\mathbb{R}^{n}\right)^{m}} \frac{1}{\left|\left(y_{1}, \ldots, y_{m}\right)\right|^{m n-\alpha}} \prod_{i=1}^{m} f_{i}\left(\ell_{i}\left(y_{1}, \ldots, y_{m}, x\right)\right) d y_{i},
$$

where $\ell_{i}$ is a linear combination of $y_{j} \mathrm{~s}$ and $x$ depending on the matrix $A$. They showed that $I_{\alpha, A}$ was of strong type $\left(L^{p_{1}} \times \cdots \times L^{p_{m}}, L^{q}\right)$ and weak type $\left(L^{p_{1}} \times \cdots \times L^{p_{m}}, L^{q, \infty}\right)$. When $\ell_{i}\left(y_{1}, \ldots, y_{m}, x\right)=x-y_{i}$, we denote this multilinear fractional type operator by $\bar{I}_{\alpha}$. In 2008 , L. Tang [20] obtained the estimation of the exponential integrability of the above operator $\bar{I}_{\alpha}$, which is quite similar to Theorem B.

Thus, it is natural to ask whether Theorem B is true or not for $I_{\Omega, \alpha}^{\Theta}$ with rough kernels. Moreover, one may ask if Theorem B still holds or not for the operator with rough kernels defined by

$$
\bar{I}_{\Omega, \alpha}(\vec{f})(x)=\int_{\left(\mathbb{R}^{n}\right)^{m}} \frac{\prod_{j=1}^{m}\left|f_{j}\left(y_{j}\right)\right|\left|\Omega_{j}\left(x-y_{j}\right)\right|}{\left|\left(x-y_{1}, x-y_{2}, \ldots, x-y_{m}\right)\right|^{m n-\alpha}} d \vec{y} .
$$

Inspired by the works above, in this paper, we study the Trudinger inequality associated to multilinear fractional integral operators $I_{\Omega, \alpha}^{\Theta}$ and $\bar{I}_{\Omega, \alpha}$ with rough homogeneous kernels. Precisely, we obtain the following theorems, which give a positive answer to the above questions.

Theorem 1.1 Let $0<\alpha<n, s=\frac{n}{\alpha}, \frac{1}{s}=\frac{1}{p_{1}}+\frac{1}{p_{2}}+\cdots+\frac{1}{p_{m}}, p_{j}>1, j=1,2, \ldots, m, m \geq 2$. Denote $B$ as a ball with radius $R$ in $\mathbb{R}^{n}$; if $f_{j} \in L_{\omega_{j}}^{p_{j}}(B), \operatorname{supp}\left(f_{j}\right) \subset B(j=1,2, \ldots, m), \Omega \in L^{n /(n-\alpha)}\left(S^{n-1}\right)$, and $v_{\vec{\omega}}=\prod_{j=1}^{m} \omega_{j}^{\frac{s}{p_{j}}}$, where $\omega_{j} \in A_{s}, \omega_{j} \geq 1$. Then for any $\gamma<1$, there is a constant $C$, independent of $n, \alpha, \theta_{j}, \gamma$, such that

$$
\int_{B} \exp \left(n \gamma\left(\frac{L I_{\Omega, \alpha}^{\Theta}(\vec{f})(x)}{\|\Omega\|_{L^{n /(n-\alpha)}} \prod_{j=1}^{m}\left\|f_{j}\right\|_{L_{\omega_{j}}^{p_{j}}}}\right)^{n /(n-\alpha)}\right) v_{\vec{\omega}} d x \leq C \prod_{j=1}^{m} \omega_{j}(B),
$$

where $L=\prod_{j=1}^{m}\left|\theta_{j}\right|^{n / p_{j}}, \Theta=\left(\theta_{1}, \theta_{2}, \ldots, \theta_{m}\right), \vec{f}=\left(f_{1}, f_{2}, \ldots, f_{K}\right)$.

Remark 1.1 If we take $\Omega=1$, then Theorem 1.1 coincides with Theorem B. If $w_{j} \equiv 1$ for $j=1, \ldots, K$, then Theorem 1.1 is just Theorem A that appeared in [6]. We give an example of $v_{\vec{\omega}}$ as follows: Let $\omega_{j}(x)=(1+|x|)^{\alpha_{j}}\left(\alpha_{j} \geq 0\right.$ for each $\left.j\right)$, then $v_{\omega}(x)$ satisfy the conditions of the above Theorem 1.1. 
Remark 1.2 Assume $m=1, \omega_{j}=1$. If $\alpha=1$, Trudinger [20] proved exponential integrability of $I_{\alpha}(\vec{f})$, and Strichartz [19] for other $\alpha$. In 1972, Hedberg [9] gave a simpler proof for all $\alpha$. In 1970, Hempel-Morris-Trudinger [10] showed that if $\gamma>1$, for $\alpha=1$ the inequality in Theorem 1.1 cannot hold, and later Adams [1] obtained the same conclusion for all $\alpha$; meanwhile, in the endpoint case $\gamma=1$, it is true. In 1985, Chang and Marshall [4] proved a similar sharp exponential inequality concerning the Dirichlet integral. Assume $m \geq 2$, $w_{j}=1$, then the result was obtained by Grafakos [7] as we have already mentioned above.

Corollary 1.2 Let $B, f_{j}, p_{j}$, s, and $v_{\vec{\omega}}$ be the same as in Theorem 1.1, then $I_{\Omega, \alpha}^{\Theta}(\vec{f})$ is in $L^{q}\left(v_{\bar{\omega}}(B)\right)$ for every $q>0$, that is,

$$
\left\|I_{\Omega, \alpha}^{\Theta}(\vec{f})\right\|_{L^{q\left(\nu_{\vec{\omega}}(B)\right)}} \leq C\|\Omega\|_{L^{n /(n-\alpha)\left(S^{n-1}\right)}} \prod_{j=1}^{m}\left\|f_{j}\right\|_{L_{\omega_{j}}^{p_{j}}}
$$

for some constant $C$ depending only on $q$ on $n$ on $\alpha$ and on the $\theta_{j}$ 's.

Theorem 1.3 Let $m \geq 2,0<\alpha<m n, 1 / p=1 / p_{1}+1 / p_{2}+\cdots+1 / p_{m}=\alpha / n$ with $1<p_{i}<\infty$ for $i=1,2, \ldots, m$. Let $B$ be a ball with radius $R$ in $\mathbb{R}^{n}$ and let $f_{j} \in L^{p_{j}}(B)$ be supported in $B$, and if $\Omega_{j}$ is homogeneous of degree zero, and $\Omega_{j} \in L^{p_{j}^{\prime}}\left(S^{n-1}\right)$, where $S^{n-1}$ denotes the sphere of $\mathbb{R}^{n}$, and $\nu_{\vec{\omega}}(\vec{y})=\prod_{j=1}^{m} \omega_{j}^{1 / p_{j}}\left(y_{j}\right)$, where $\vec{y}=\left(y_{1}, y_{2}, \ldots, y_{m}\right)$ and $\omega_{j} \in A_{s}, \omega_{j} \geq 1$. Then there exist constants $k_{1}, k_{2}$ depending only on $n, m, \alpha, p$, and the $p_{j}$ such that

$$
\int_{B} \exp \left(k_{1}\left(\frac{\left|\bar{I}_{\Omega, \alpha}(\vec{f})(x)\right|}{\prod_{j=1}^{m}\left\|\Omega_{j}\right\|_{L^{p_{j}^{\prime}}\left(S^{n-1}\right)}\left\|f_{j}\right\|_{L_{\omega_{j}}^{p_{j}}}}\right)^{n /(m n-\alpha)}\right) v_{\vec{\omega}}(x) d x \leq k_{2} \prod_{j=1}^{m} \omega_{j}(B) .
$$

Remark 1.3 If we take $\Omega=1, w_{j} \equiv 1$ for $j=1, \ldots, m$, then Theorem 1.3 is just as Theorem 1.3 appeared in [20]. But there is something that needs to be changed in the proof of Theorem 1.3 in [20]. In the case $r_{1}=r_{2}=\cdots=r_{m-1}=0$, one cannot obtain the conclusion that $F_{2} \leq C_{2}\left[\log \frac{2 \sqrt{m} R}{\delta}\right]^{(m n-\alpha) / n}$. Thus, our proof gives an alternative correction of Theorem 1.3 in [20].

Corollary 1.4 Let $B, f_{j}, p_{j}$, s, and $v_{\vec{\omega}}$ be the same as in Theorem 1.3. Then $\bar{I}_{\Omega, \alpha}(\vec{f})$ is in $L^{q}\left(v_{\vec{\omega}}(B)\right)$ for every $q>0$, that is,

$$
\left\|\bar{I}_{\Omega, \alpha}(\vec{f})\right\|_{L^{q}\left(\vec{v}_{\bar{\omega}}(B)\right)} \leq C \prod_{j=1}^{m}\left\|\Omega_{j}\right\|_{L^{p_{j}^{\prime}\left(S^{n-1}\right)}}\left\|f_{j}\right\|_{L_{\omega_{j}}^{p_{j}}}
$$

for some constant $C$ depending only on $q$ on $n$ on $\alpha$.

Corollary 1.2 and Corollary 1.4 follow since exponential integrability of $\bar{I}_{\Omega, \alpha}(\vec{f})$ implies integrability to any power $q$.

On the other hand, we shall study the boundedness of the multilinear fractional maximal operator with a weighted norm. It follows the following theorem. 
Theorem 1.5 If $1<p_{j}<\infty, \frac{1}{s}=\sum_{j=1}^{m} \frac{1}{p_{j}}, \frac{1}{r}=\frac{1}{s}-\frac{\alpha}{n}, \omega_{j}^{\frac{p_{j}}{s}} \in A\left(s, \frac{s r_{j}}{p_{j}}\right), 1 / r_{j}=1 / p_{j}(1-\alpha s / n)$, $j=1,2, \ldots, m, v_{\vec{\omega}}=\prod_{j=1}^{m} \omega_{j}$, then there is a constant $C$, independent $f_{j}$, such that

$$
\left(\int_{\mathbb{R}^{n}}\left(M_{1, \alpha}^{\Theta}(\vec{f})(x) v_{\vec{\omega}}(x)\right)^{r} d x\right)^{\frac{1}{r}} \leq C \prod_{j=1}^{m}\left(\int_{\mathbb{R}^{n}}\left|f_{j}(x) \omega_{j}(x)\right|^{p_{j}} d x\right)^{\frac{1}{p_{j}}},
$$

where $\vec{f}=\left(f_{1}, f_{2}, \ldots, f_{m}\right), f_{j} \in L_{\omega_{j}}^{p_{j}}\left(\mathbb{R}^{n}\right)$.

\section{The proof of Theorem 1.1}

In this section, we will prove Theorem 1.1.

Proof For any $\delta>0$,

$$
\left|I_{\Omega, \alpha}^{\Theta}(\vec{f})(x)\right| \leq C \delta^{\alpha} M_{\Omega}(\vec{f})(x)+\int_{|y| \geq \delta} \frac{|\Omega(y)|}{|y|^{n-\alpha}} \prod_{j=1}^{m} f_{j}\left(x-\theta_{j} y\right) d y .
$$

Set $P=2 \min \left\{\frac{1}{\theta_{j}}: j=1,2, \ldots, K\right\}$. For any $R>0$, denote $B(R)$ as a ball with radius $R$ in $\mathbb{R}^{n}$, then for any $x \in B(R)$, when $\left|x-\theta_{j} y\right|<R,\left|\theta_{j} y\right|<2 R$ for $j=1, \ldots, m$. Therefore, $|y|<R P$. So,

$$
\int_{|y| \geq \delta} \prod_{j=1}^{m} f_{j}\left(x-\theta_{j} y\right)|y|^{\alpha-n} d y=\int_{\delta \leq|y|<P R} \prod_{j=1}^{m} f_{j}\left(x-\theta_{j} y\right)|y|^{\alpha-n} d y .
$$

According to the relationship between $s$ and $p_{j}: \frac{1}{p_{1}}+\frac{1}{p_{2}}+\cdots+\frac{1}{p_{m}}+\frac{1}{n /(n-\alpha)}=1$, from the Hölder's inequality and $v_{\vec{\omega}} \geq 1$, it follows that

$$
\begin{aligned}
& \int_{\delta \leq|y|<P R} \Omega(y) \prod_{j=1}^{m} f_{j}\left(x-\theta_{j} y\right)|y|^{\alpha-n} d y \\
& \quad \leq\left(\int_{\delta \leq|y| \leq P R}\left(\prod_{j=1}^{m} f_{j}\left(x-\theta_{j} y\right)\right)^{s} d y\right)^{1 / s}\left(\int_{\delta \leq|y| \leq P R}\left(\frac{|\Omega(y)|}{|y|^{n-\alpha}}\right)^{s^{\prime}} d y\right)^{1 / s^{\prime}} \\
& \quad \leq\left(\int_{\delta \leq|y| \leq P R} \prod_{j=1}^{m} f_{j}\left(x-\theta_{j} y\right)^{s} v_{\bar{\omega}}\left(x-\theta_{j} y\right) d y\right)^{1 / s}\|\Omega\|_{L^{s^{\prime}}}\left(\ln \frac{P R}{\delta}\right)^{\frac{n-\alpha}{n}} \\
& \quad \leq \prod_{j=1}^{m}\left(\int_{\delta \leq|y| \leq P R}\left|f_{j}\left(x-\theta_{j} y\right)\right|^{p_{j}} \omega_{j}\left(x-\theta_{j} y\right) d y\right)^{\frac{1}{p_{j}}}\|\Omega\|_{L^{s^{\prime}}}\left(\frac{1}{n} \ln \left(\frac{P R}{\delta}\right)^{n}\right)^{\frac{n-\alpha}{n}} \\
& \quad \leq L^{-1} \prod_{j=1}^{m}\left\|f_{j}\right\|_{L_{\omega_{j}}} p_{j}\|\Omega\|_{L^{s^{\prime}}}\left(\frac{1}{n} \ln \left(\frac{P R}{\delta}\right)^{n}\right)^{\frac{n-\alpha}{n}} .
\end{aligned}
$$

Hence, we obtain that

$$
\left|I_{\Omega, \alpha}^{\Theta}(\vec{f})(x)\right| \leq C \delta^{\alpha} M_{\Omega} \vec{f}(x)+L^{-1} \prod_{j=1}^{m}\left\|f_{j}\right\|_{L_{\omega_{j}} p_{j}}\|\Omega\|_{L^{s^{\prime}}}\left(\frac{1}{n} \ln \left(\frac{P R}{\delta}\right)^{n}\right)^{\frac{n-\alpha}{n}}
$$


Set $\delta=\varepsilon\left(\left|I_{\Omega, \alpha}^{\Theta}(\vec{f})(x)\right| / C M_{\Omega}(\vec{f})(x)\right)^{1 / \alpha}$, then

$$
\exp \left\{n \gamma\left(\frac{L I_{\Omega, \alpha}^{\Theta}(\vec{f})(x)}{\|\Omega\|_{L^{s}} \prod_{j=1}^{m}\left\|f_{j}\right\|_{L_{\omega_{j}}}}\right)^{\frac{n}{n-\alpha}}\right\} \leq \ln C R^{n}\left(\frac{M_{\Omega}(\vec{f})(x)}{I_{\Omega, \alpha}^{\Theta}(\vec{f})(x)}\right)^{n / \alpha} .
$$

Now we put $B_{1}=\left\{x \in B: \frac{I_{\Omega, \alpha}^{\Theta}(\vec{f})(x)}{\|\Omega\|_{L^{n /(n-\alpha)}}^{m} \prod_{j=1}^{m}\left\|f_{j}\right\|_{L_{\omega_{j}}}} \geq 1\right\}, B_{2}=B-B_{1}$, thus

$$
\begin{aligned}
& \int_{B_{1}} \exp \left(n \gamma\left(\frac{L I_{\Omega, \alpha}^{\Theta}(\vec{f})(x)}{\|\Omega\|_{L^{n /(n-\alpha)}} \prod_{j=1}^{m}\left\|f_{j}\right\|_{L_{\omega_{j}}^{p_{j}}}}\right)^{n /(n-\alpha)}\right) v_{\vec{\omega}}(x) d x \\
& \leq C R^{n} \int_{B_{1}}\left(\frac{M_{\Omega}(\vec{f})(x)}{I_{\Omega, \alpha}^{\Theta}(\vec{f})(x)}\right)^{n / \alpha} v_{\vec{\omega}}(x) d x \\
& \leq C R^{n} \int_{B_{1}}\left(\frac{M_{\Omega}(\vec{f})(x)}{\|\Omega\|_{L^{n /(n-\alpha)}} \prod_{j=1}^{m}\left\|f_{j}\right\|_{L_{\omega_{j}}^{p_{j}}}}\right)^{n / \alpha} v_{\bar{\omega}}(x) d x .
\end{aligned}
$$

By the fact that

$$
\begin{aligned}
& M_{\Omega}(\vec{f})(x)=\sup _{r>0} \int_{|y|<r}|\Omega(y)|^{\sum_{j=1}^{m} \frac{s}{p_{j}}} \prod_{j=1}^{m} f_{j}\left(x-\theta_{j} y\right) d y \\
& \leq \sup _{r>0} \prod_{j=1}^{m}\left(\frac{1}{r^{n}} \int_{|y|<r}|\Omega(y)| f_{j}^{\frac{p_{j}}{s}}\left(x-\theta_{j} y\right) d y\right)^{\frac{s}{p_{j}}} \\
& \leq \prod_{j=1}^{m}\left(M_{\Omega}\left(f^{\frac{p_{j}}{s}}\right)(x)\right)^{\frac{s}{p_{j}}} .
\end{aligned}
$$

Therefore, we get

$$
\begin{aligned}
& \int_{B_{1}} \exp \left(n \gamma\left(\frac{L I_{\Omega, \alpha}^{\Theta}(\vec{f})(x)}{\|\Omega\|_{L^{n /(n-\alpha)}} \prod_{j=1}^{m}\left\|f_{j}\right\|_{L_{\omega_{j}}^{p_{j}}}}\right)^{n /(n-\alpha)}\right) v_{\vec{\omega}}(x) d x \\
& \leq \frac{C R^{n}}{\|\Omega\|_{L^{n /(n-\alpha)}} \prod_{j=1}^{m}\left\|f_{j}\right\|_{L_{\omega_{j}}^{s}}^{s}} \int_{B_{1}} \prod_{j=1}^{m}\left(M_{\Omega}\left(f_{j}^{\frac{p_{j}}{s}}(x)\right)\right)^{\frac{s^{2}}{p_{j}}} v_{\vec{\omega}}(x) d x \\
& \leq \frac{C R^{n}}{\|\Omega\|_{L^{n /(n-\alpha)}} \prod_{j=1}^{m}\left\|f_{j}\right\|_{L_{\omega_{j}}^{p-j}}^{s}} \prod_{j=1}^{m}\left(\int_{B_{1}}\left(M_{\Omega}\left(f_{j}^{\frac{p_{j}}{s}}(x)\right)\right)^{s} \omega_{j}(x) d x\right)^{\frac{1}{s} s^{2}} \\
& \leq \frac{C R^{n}}{\|\Omega\|_{L^{n /(n-\alpha)}} \prod_{j=1}^{m}\left\|f_{j}\right\|_{L_{\omega_{j}}^{s}}^{s}} \prod_{j=1}^{m}\left\|f_{j}^{\frac{p_{j}}{s}}\right\|_{L_{\omega_{j}}^{p^{s}}}^{\frac{s^{2}}{p_{j}}} \\
& \leq C R^{n} .
\end{aligned}
$$

Here, in the above third inequality, we have used the well-known weighted result of HardyLittlewood maximal function. 
Fens and Xe Journal of Inequalities and Applications 2012, 2012:179

Page 7 of 12

http://www.journalofinequalitiesandapplications.com/content/2012/1/179

From $\omega_{j} \geq 1(j=1,2, \ldots, m)$, we get

$$
R^{n}=c \int_{B} d x \leq c \int_{B} \omega_{j}(x) d x=c \omega_{j}(B) .
$$

Hence,

$$
\int_{B_{1}} \exp \left(n \gamma\left(\frac{L I_{\Omega, \alpha}^{\Theta}(\vec{f})(x)}{\|\Omega\|_{L^{n /(n-\alpha)}} \prod_{j=1}^{m}\left\|f_{j}\right\|_{L_{\omega_{j}} p_{j}}}\right)^{n /(n-\alpha)}\right) v_{\vec{\omega}}(x) d x \leq C^{\prime} \prod_{j=1}^{m} \omega_{j}(B) .
$$

On the other hand,

$$
\begin{aligned}
& \int_{B_{2}} \exp \left(n \gamma\left(\frac{L I_{\Omega, \alpha}^{\Theta}(\vec{f})(x)}{\|\Omega\|_{L^{n /(n-\alpha)}} \prod_{j=1}^{m}\left\|f_{j}\right\|_{L_{\omega_{j}}}}\right)^{n /(n-\alpha)}\right) v_{\vec{\omega}}(x) d x \\
& \quad \leq \exp (n \gamma)\left(\frac{L}{\|\Omega\|_{L^{s^{\prime}}}}\right)^{\frac{n}{n-\alpha}} \int_{B_{2}} v_{\vec{\omega}}(x) d x \\
& \quad \leq C \prod_{j=1}^{m} \omega_{j}(B) .
\end{aligned}
$$

From the above all, we obtain that

$$
\int_{B} \exp \left(n \gamma\left(\frac{L I_{\Omega, \alpha}^{\Theta}(\vec{f})(x)}{\|\Omega\|_{L^{n /(n-\alpha)}} \prod_{j=1}^{m}\left\|f_{j}\right\|_{L_{\omega_{j}}}}\right)^{n /(n-\alpha)}\right) v_{\vec{\omega}}(x) d x \leq C \prod_{j=1}^{m} \omega_{j}(B) .
$$

3 The proof of Theorem 1.5

In this section, we will prove Theorem 1.5.

Proof By the well-known Hölder's inequality, we get

$$
\begin{aligned}
M_{1, \alpha}(\vec{f})(x) & =\sup _{r>0} \frac{1}{|r|^{n-\alpha}} \int_{|y|<r} \prod_{j=1}^{m} f_{j}(x-y) d y \\
& \leq \sup _{r>0} \frac{1}{|r|^{n-\alpha}} \prod_{j=1}^{m}\left(\int_{|y|<r} f_{j}^{\frac{p_{j}}{s}}(x-y) d y\right)^{\frac{s}{p_{j}}} \\
& \leq \prod_{j=1}^{m}\left(\sup _{r>0} \frac{1}{|r|^{n-\alpha}} \int_{|y|<r} f_{j}^{\frac{p_{j}}{s}}(x-y) d y\right)^{\frac{s}{p_{j}}} \\
& =\prod_{j=1}^{m}\left(M_{1, \alpha}\left(f^{p_{j} / s}\right)(x)\right)^{\frac{s}{p_{j}}} .
\end{aligned}
$$

Hence,

$$
\begin{aligned}
\left(\int_{\mathbb{R}^{n}}\left(M_{1, \alpha}(\vec{f})(x) v_{\vec{\omega}}(x)\right)^{r} d x\right)^{1 / r} & \leq\left[\int_{\mathbb{R}^{n}}\left(\prod_{j=1}^{m}\left[M_{1, \alpha}\left(f^{p_{j} / s}\right)(x) \omega_{j}^{p_{j} / s}(s)\right]^{\frac{s}{p_{j}}}\right)^{r} d x\right]^{1 / r} \\
& \leq \prod_{j=1}^{m}\left[\int_{\mathbb{R}^{n}}\left(M_{1, \alpha}\left(f_{j}^{p_{j} / s}\right)(x) \omega^{p_{j} / s}(x)\right)^{s r_{j} / p_{j}} d x\right]^{\frac{p_{j}}{s r_{j}} \frac{s}{p_{j}}} .
\end{aligned}
$$


In addition, from the condition $\omega_{j}^{p_{j} / s}(x) \in A\left(s, \frac{s r_{j}}{p_{j}}\right)$, it follows that

$$
\left[\int_{\mathbb{R}^{n}}\left(M_{1, \alpha}\left(f_{j}^{p_{j} / s}\right)(x) \omega^{p_{j} / s}(x)\right)^{s r_{j} / p_{j}} d x\right]^{\frac{p_{j}}{s r_{j}} \frac{s}{p_{j}}} \leq C_{j}\left[\int_{\mathbb{R}^{n}}\left(f_{j}^{p_{j} / s}(x) \omega_{j}^{p_{j} / s}(x)\right)^{s} d x\right]^{1 / p_{j}} .
$$

According to the above, we obtain that

$$
\left(\int_{R^{n}}\left(M_{1, \alpha}(\vec{f})(x) v_{\vec{\omega}}(x)\right)^{r} d x\right)^{1 / r}=C \prod_{j=1}^{m}\left(\int_{\mathbb{R}^{n}}\left(f_{j}(x) \omega_{j}(x)\right)^{p_{j}} d x\right)^{1 / p_{j}} .
$$

It is easy to see that

$$
M_{1, \alpha}^{\Theta}(\vec{f})(x)=\sup _{r>0} \frac{1}{r^{n-\alpha}} \int_{|y|<r} \prod_{j=1}^{m}\left|f_{j}\left(x-\theta_{j} y\right)\right| d y,
$$

where $\Theta=\left(\theta_{1}, \theta_{2}, \ldots, \theta_{m}\right), \theta_{j} \in \mathbb{R}$ holds, also.

\section{The proof of Theorem 1.3}

In this section, we will prove Theorem 1.3.

Proof For any $\delta>0$ and $x \in B$,

$$
\begin{aligned}
& \left|\bar{I}_{\Omega, \alpha}\left(f_{1}, f_{2}, \ldots, f_{m}\right)(x)\right| \\
& \leq \int_{\left|\left(x-y_{1}, x-y_{2}, \ldots, x-y_{m}\right)\right|<\delta} \frac{\prod_{j=1}^{m}\left|\Omega_{j}\left(y_{j}\right) f_{j}\left(y_{j}\right)\right|}{\left|\left(x-y_{1}, x-y_{2}, \ldots, x-y_{m}\right)\right|^{m n-\alpha}} d \vec{y} \\
& \quad+\int_{\left|\left(x-y_{1}, x-y_{2}, \ldots, x-y_{m}\right)\right| \geq \delta} \frac{\prod_{j=1}^{m}\left|\Omega_{j}\left(y_{j}\right) f_{j}\left(y_{j}\right)\right|}{\left|\left(x-y_{1}, x-y_{2}, \ldots, x-y_{m}\right)\right|^{m n-\alpha}} d \vec{y} \\
& \quad:=F_{1}+F_{2} .
\end{aligned}
$$

For $F_{1}$, let $\alpha=\sum_{j=1}^{m} \alpha_{j}$ with $\alpha_{j}=n / p_{j}$ for $j=1,2, \ldots, m$. Then

$$
\begin{aligned}
F_{1} & \leq \int_{\left|\left(x-y_{1}, x-y_{2}, \ldots, x-y_{m}\right)\right|<\delta} \frac{\left|\Omega_{j}\left(y_{j}\right) f_{j}\left(y_{j}\right)\right|}{\prod_{j=1}^{m}\left|x-y_{j}\right|^{n-\alpha_{j}}} d \vec{y} \\
& \leq \prod_{j=1}^{m} \int_{\left|x-y_{j}\right|<\delta} \frac{\left|\Omega_{j}\left(y_{j}\right) f_{j}\left(y_{j}\right)\right|}{\left|x-y_{j}\right|^{n-\alpha_{j}}} d y_{j} \\
& \leq C \prod_{j=1}^{m} \delta^{\alpha_{j}} M_{\Omega_{j}}\left(f_{j}\right)(x) \\
& :=C_{1} \delta^{\alpha} \prod_{j=1}^{m} M_{\Omega_{j}}\left(f_{j}\right)(x),
\end{aligned}
$$

where $M_{\Omega}$ denotes as $M_{\Omega}(f)(x)=\sup _{r>0} \frac{1}{r^{n}} \int_{|x-y|<r}|\Omega(y) f(y)| d y$.

For $F_{2}$, if $\left(y_{1}, y_{2}, \ldots, y_{m}\right)$ satisfies $\left|\left(x-y_{1}, x-y_{2}, \ldots, x-y_{m}\right)\right| \geq \delta$, then for some $j \in 1,2, \ldots, m$, $\left|x-y_{j}\right| \leq \frac{\delta}{\sqrt{m}}$. Without losing the generalization, we set $j=m$. 
Thus,

$$
F_{2} \leq \int_{\delta / \sqrt{m} \leq\left|x-y_{m}\right| \leq 2 R} \int_{\left(\mathbb{R}^{n}\right)^{m-1}} \frac{\prod_{j=1}^{m}\left|\Omega_{j}\left(y_{j}\right) f_{j}\left(y_{j}\right)\right|}{\left|\left(x-y_{1}, x-y_{2}, \ldots, x-y_{m}\right)\right|^{m n-\alpha}} d \vec{y} .
$$

Define that $f_{j}^{0}=f_{j} \chi_{B(x, \delta / \sqrt{m})}$ and $f_{j}^{\infty}=f-f_{j}^{0}$ for $j=1,2, \ldots, m$. By the condition of $v_{\vec{\omega}}$, we have

$$
\begin{aligned}
F_{2} & \leq \sum_{\vec{r} \in\{0, \infty\}^{m}} \int_{\delta / \sqrt{m} \leq\left|x-y_{m}\right| \leq 2 R} \int_{\left(\mathbb{R}^{n}\right)^{m-1}} \frac{\prod_{j=1}^{m-1}\left|\Omega_{j}\left(y_{j}\right) f_{j}^{r_{j}}\left(y_{j}\right)\right|\left|\Omega_{m}\left(y_{m}\right) f_{m}\left(y_{m}\right)\right|}{\left|\left(x-y_{1}, x-y_{2}, \ldots, x-y_{m}\right)\right|^{m n-\alpha}} d \vec{y} \\
& \leq \sum_{\vec{r} \in\{0, \infty\}^{m}} \int_{\delta / \sqrt{m} \leq\left|x-y_{m}\right| \leq 2 R} \int_{\left(\mathbb{R}^{n}\right)^{m-1}} \frac{\prod_{j=1}^{m-1}\left|\Omega_{j}\left(y_{j}\right) f_{j}^{r_{j}}\left(y_{j}\right)\right|\left|\Omega_{m}\left(y_{m}\right) f_{m}\left(y_{m}\right)\right|}{\left|\left(x-y_{1}, x-y_{2}, \ldots, x-y_{m}\right)\right|^{m n-\alpha}} v_{\vec{\omega}}(\vec{y}) d \vec{y},
\end{aligned}
$$

where $\vec{r}=\left(r_{1}, r_{2}, \ldots, r_{m}\right)$. In the case that $r_{1}=r_{2}=\cdots=r_{m-1}=0$, by the fact that

$$
\begin{aligned}
\left|\left(x-y_{1}, x-y_{2}, \ldots, x-y_{m}\right)\right|^{m n-\alpha} & \geq\left|x-y_{m}\right|^{m n-\alpha} \\
& =\left|x-y_{m}\right|^{n-\alpha_{m}}\left|x-y_{m}\right|^{\sum_{j=1}^{m-1} n \mid p_{j}^{\prime}} \\
& \geq\left|x-y_{m}\right|^{n-\alpha_{m}}\left(\frac{\delta}{\sqrt{m}}\right)^{\sum_{j=1}^{m-1} n / p_{j}^{\prime}},
\end{aligned}
$$

we have

$$
\begin{aligned}
\int_{\delta / \sqrt{m} \leq\left|x-y_{m}\right| \leq 2 R} & \int_{\left(\mathbb{R}^{n}\right)^{m-1}} \frac{\prod_{j=1}^{m-1}\left|\Omega_{j}\left(y_{j}\right) f_{j}^{0}\left(y_{j}\right)\right|\left|\Omega\left(y_{m}\right) f_{m}\left(y_{m}\right)\right|}{\left|\left(x-y_{1}, x-y_{2}, \ldots, x-y_{m}\right)\right|^{m n-\alpha}} v_{\vec{\omega}}(\vec{y}) d \vec{y} \\
\leq & \prod_{j=1}^{m-1} \delta^{-\frac{n}{p_{j}^{\prime}}} \int_{\frac{\delta}{\sqrt{m}} \leq\left|x-y_{m}\right| \leq 2 R} \frac{\left|\Omega_{m}\left(y_{m}\right) f_{m}\left(y_{m}\right)\right|}{\left|x-y_{m}\right|^{n-\alpha_{m}}} \omega_{m}^{1 / p_{m}}\left(y_{m}\right) d y_{m} \\
& \times \prod_{j=1}^{m-1} \int_{\left|x-y_{j}\right|<\delta / \sqrt{m}}\left|\Omega_{j}\left(y_{j}\right) f_{j}\left(y_{j}\right)\right| \omega_{j}^{1 / p_{j}}\left(y_{j}\right) d y_{j} \\
\leq C & \prod_{j=1}^{m}\left\|\Omega_{j}\right\|_{L^{p_{j}^{\prime}\left(S^{n-1}\right)}}\left\|f_{j}\right\|_{L_{\omega_{j}}}\left(\log \frac{2 R \sqrt{m}}{\delta}\right)^{1 / p_{m}^{\prime}} \\
\leq C & \prod_{j=1}^{m}\left\|\Omega_{j}\right\|_{L^{p_{j}^{\prime}}}\left\|f_{\left.S^{n-1}\right)}\right\|_{L_{\omega_{j}} p_{j}}\left(\log \frac{2 R \sqrt{m}}{\delta}\right)^{(m n-\alpha) / n} .
\end{aligned}
$$

Consider the case where exactly $l$ of the $r_{j}$ are $\infty$ for some $1 \leq l \leq m$. Without losing the generalization, we only give the argument for $r_{j}=\infty, j=1,2, \ldots, l$, then

$$
\begin{aligned}
& \int_{\delta / \sqrt{m} \leq\left|x-y_{m}\right| \leq 2 R} \int_{\left(\mathbb{R}^{n}\right)^{m-1}} \frac{\prod_{j=1}^{m} \Omega_{j}\left(y_{j}\right) \prod_{j=1}^{l}\left|f_{j}^{\infty}\left(y_{j}\right) \prod_{k=l+1}^{m-1} f_{k}^{0}\left(y_{k}\right) f_{m}\left(y_{m}\right)\right|}{\left|\left(x-y_{1}, x-y_{2}, \ldots, x-y_{m}\right)\right|^{m n-\alpha}} v_{\vec{\omega}} d \vec{y} \\
& \leq \prod_{k=l+1}^{m-1} \int_{\left|x-y_{k}\right|<\delta / \sqrt{m}}\left|\Omega_{k}\left(y_{k}\right) f_{k}\left(y_{k}\right)\right| \omega_{k}^{1 / p_{m}}\left(y_{k}\right) d y_{k} \\
& \quad \times \prod_{j=1}^{l} \int_{\delta / \sqrt{m} \leq\left|x-y_{j}\right| \leq 2 R} \frac{\left|\Omega_{j}\left(y_{j}\right) f_{j}\left(y_{j}\right)\right|}{\left|x-y_{j}\right|^{n-\alpha_{j}}} \omega_{j}^{1 / p_{j}}\left(y_{j}\right) d y_{j}
\end{aligned}
$$


Fens and Xe Journal of Inequalities and Applications 2012, 2012:179

Page 10 of 12

http://www.journalofinequalitiesandapplications.com/content/2012/1/179

$$
\begin{aligned}
& \times \int_{\delta / \sqrt{m} \leq\left|x-y_{m}\right| \leq 2 R} \frac{\left|\Omega_{m}\left(y_{m}\right) f_{m}\left(y_{m}\right)\right|}{\left|x-y_{m}\right|^{(m-l) n-\sum_{k=l+1}^{m} \alpha_{k}}} \omega_{m}^{1 / p_{m}}\left(y_{m}\right) d y_{m} \\
\leq & C\left[\log \frac{2 \sqrt{m} R}{\delta}\right]^{\sum_{k=1}^{l} \frac{1}{p_{m}^{\prime}}} \prod_{j=1}^{m}\left\|\Omega_{j}\right\|_{L_{j}^{p_{j}^{\prime}\left(S^{n-1}\right)}}\left\|f_{j}\right\|_{L_{\omega_{j}}^{p_{j}}} \\
\leq & C \prod_{j=1}^{m}\left\|\Omega_{j}\right\|_{L^{p_{j}^{\prime}\left(S^{n-1}\right)}}\left\|f_{j}\right\|_{L_{\omega_{j}}}\left[\log \frac{2 \sqrt{m} R}{\delta}\right]^{(m n-\alpha) / n} .
\end{aligned}
$$

Combining the above cases, we obtain

$$
F_{2} \leq C_{2} \prod_{j=1}^{m}\left\|\Omega_{j}\right\|_{L^{p_{j}^{\prime}\left(S^{n-1}\right)}}\left\|f_{j}\right\|_{L_{\omega_{j}}}\left[\log \frac{2 \sqrt{m} R}{\delta}\right]^{(m n-\alpha) / n} .
$$

Thus, by the estimates for $F_{1}, F_{2}$, we have

$$
\begin{aligned}
\bar{I}_{\Omega, \alpha}\left(f_{1}, f_{2}, \ldots, f_{m}\right)(x) \leq & C_{1} \delta^{\alpha} \prod_{j=1}^{m} M_{\Omega_{j}}\left(f_{j}\right)(x) \\
& +C_{2} \prod_{j=1}^{m}\left\|\Omega_{j}\right\|_{L^{p_{j}^{\prime}\left(S^{n-1}\right)}}\left\|f_{j}\right\|_{L_{\omega_{j}}^{p_{j}}}\left[\log \frac{2 \sqrt{m} R}{\delta}\right]^{(m n-\alpha) / n} .
\end{aligned}
$$

In particular, we chose $\delta=2 \sqrt{m} R$ for all $x \in B$, then

$$
\bar{I}_{\Omega, \alpha}\left(f_{1}, f_{2}, \ldots, f_{m}\right)(x) \leq C_{1} \delta^{\alpha} \prod_{j=1}^{m} M_{\Omega_{j}}\left(f_{j}\right)(x)
$$

Now, we set

$$
\delta=\delta(x)=\varepsilon\left[\left|\bar{I}_{\Omega, \alpha}\left(f_{1}, f_{2}, \ldots, f_{m}\right)(x)\right| / C_{1} \prod_{j=1}^{m} M_{\Omega_{j}}\left(f_{j}\right)(x)\right]^{1 / \alpha}
$$

where $\varepsilon<1$.

Then

$$
\begin{aligned}
& \left|\bar{I}_{\Omega, \alpha}\left(f_{1}, f_{2}, \ldots, f_{m}\right)(x)\right| \\
& \leq \varepsilon^{\alpha}\left|\bar{I}_{\Omega, \alpha}\left(f_{1}, f_{2}, \ldots, f_{m}\right)(x)\right| \\
& \quad+C_{2} \prod_{j=1}^{m}\left\|\Omega_{j}\right\|_{L^{p_{j}^{\prime}\left(S^{n-1}\right)}}\left\|f_{j}\right\|_{L_{\omega_{j}}^{p_{j}}}\left[\frac{1}{n} \log \left(\frac{(2 \sqrt{m} R)^{n}\left[C_{1} \prod_{j=1}^{m} M_{\Omega_{j}}\left(f_{j}\right)(x)\right]^{n / \alpha}}{\varepsilon^{n}\left|\bar{I}_{\Omega, \alpha}\left(f_{1}, f_{2}, \ldots, f_{m}\right)(x)\right|^{n / \alpha}}\right)\right]^{(m n-\alpha) / n} .
\end{aligned}
$$

Hence,

$$
\exp \left(k_{1}\left(\frac{\left|\bar{I}_{\Omega, \alpha}\left(f_{1}, f_{2}, \ldots, f_{m}\right)(x)\right|}{\prod_{j=1}^{m}\left\|\Omega_{j}\right\|_{L^{p_{j}^{\prime}\left(S^{n-1}\right)}}\left\|f_{j}\right\|_{L_{\omega_{j}}^{p_{j}}}}\right)^{n /(m n-\alpha)}\right) \leq \frac{C\left[\prod_{j=1}^{m} M_{\Omega_{j}}\left(f_{j}\right)(x)\right]^{n / \alpha}}{\left|\bar{I}_{\Omega, \alpha}\left(f_{1}, f_{2}, \ldots, f_{m}\right)(x)\right|^{n / \alpha}}
$$


Let $B_{1}=\left\{x \in B: \frac{\left|\bar{I}_{\Omega, \alpha}\left(f_{1}, f_{2}, \ldots, f_{m}\right)(x)\right|}{\prod_{j=1}^{m}\left\|\Omega_{j}\right\|_{L} p_{j}^{\prime}\left\|f_{\left(S^{n-1}\right)}\right\|_{L} p_{j}} \geq 1\right\}$ and $B_{2}=B-B_{1}$, then

$$
\begin{aligned}
& \int_{B_{1}} \exp \left(k_{1}\left(\frac{\left|\bar{I}_{\Omega, \alpha}\left(f_{1}, f_{2}, \ldots, f_{m}\right)(x)\right|}{\prod_{j=1}^{m}\left\|\Omega_{j}\right\|_{L^{p_{j}^{\prime}}\left(S^{n-1}\right)}\left\|f_{j}\right\|_{L_{\omega_{j}}^{p_{j}}}}\right)^{n /(m n-\alpha)}\right) v_{\vec{\omega}} d x \\
& \leq C R^{n} \int_{B_{1}}\left(\frac{\prod_{j=1}^{m} M_{\Omega_{j}}\left(f_{j}\right)(x)}{\prod_{j=1}^{m}\left\|\Omega_{j}\right\|_{\left.L_{j}^{p_{j}^{\prime}} S^{n-1}\right)}\left\|f_{j}\right\|_{L_{\omega_{j}}^{p_{j}}}}\right)^{n / \alpha} v_{\vec{\omega}} d x \\
& \leq C R^{n}\left(\prod_{j=1}^{m} \frac{\left\|M_{\Omega_{j}}\left(f_{j}\right)\right\|_{L_{\omega_{j}}^{p_{j}}}}{\left\|\Omega_{j}\right\|_{L_{j_{(S-1}}^{\prime}}\left\|f_{j}\right\|_{L_{\omega_{j}}}}\right)^{n / \alpha} \\
& \leq C R^{n} \\
& \leq C \prod_{j}^{m} \omega_{j}(B) .
\end{aligned}
$$

On the other hand,

$$
\begin{aligned}
& \int_{B_{2}} \exp \left(k_{1}\left(\frac{\left|\bar{I}_{\Omega, \alpha}\left(f_{1}, f_{2}, \ldots, f_{m}\right)(x)\right|}{\prod_{j=1}^{m}\left\|\Omega_{j}\right\|_{L^{p_{j}^{\prime}\left(S^{n-1}\right)}}\left\|f_{j}\right\|_{L^{p_{j}}}}\right)^{n /(m n-\alpha)}\right) v_{\vec{\omega}}(x) d x \\
& \leq \exp \left(k_{1}\right) \prod_{j=1}^{m} \int_{B_{2}} \omega_{j}(x) d x \\
& \leq C \prod_{j=1}^{m} \omega_{j}(B)
\end{aligned}
$$

Combining the above results, we obtain

$$
\int_{B} \exp \left(k_{1}\left(\frac{\left|\bar{I}_{\Omega, \alpha}\left(f_{1}, f_{2}, \ldots, f_{m}\right)(x)\right|}{\prod_{j=1}^{m}\left\|\Omega_{j}\right\|_{L^{p_{j}^{\prime}}\left(S^{n-1}\right)}\left\|f_{j}\right\|_{L^{p_{j}}}}\right)^{n /(m n-\alpha)}\right) v_{\vec{\omega}}(x) d x \leq k_{2} \prod_{j=1}^{m} \omega_{j}(B)
$$

where $k_{1}, k_{2}$ are constants depending only on $n, m, \alpha, p$, and the $p_{j}$.

\section{Competing interests}

The authors declare that they have no competing interests.

Authors' contributions

All authors read and approved the final manuscript.

\section{Authors' information}

H. Feng's current address: Department of Mathematical and Statistical Sciences, University of Alberta, Canada.

\section{Acknowledgement}

The second author was supported partly by NSFC (Grant No. 10701010), NSFC (Key program Grant No. 10931001), Beijing Natural Science Foundation (Grant: 1102023), Program for Changjiang Scholars and Innovative Research Team in University. 


\section{References}

1. Adams, D: A sharp inequality of J. Moser for higher order derivatives. Ann. Math. 128, 385-398 (1988)

2. Cerny, R, Gurka, P, Hencl, S: Concentration compactness principle for generalized Trudinger inequalities. Z. Anal. Anwend. 30(3), 355-375 (2011)

3. Carleson, L, Chang, S: On the existence of an extremal function for an inequality of J. Moser. Bull. Sci. Math. (2) 110(2), 113-127 (1986)

4. Chang, S, Marshall, D: On a sharp inequality concerning the Dirichlet integral. Am. J. Math. 107, 1015-1033 (1985)

5. Chen, X, Xue, Q: Weighted estimates for a class of multilinear fractional type operators. J. Math. Anal. Appl. 362(2), 355-373 (2010)

6. Ding, Y, Lu, S: The $L^{p_{1}} \times L^{p_{2}} \times \cdots \times L^{p_{m}}$ boundedness for some rough operators. J. Math. Anal. Appl. 203, 166-186 (1996)

7. Grafakos, L: On multilinear fractional integrals. Stud. Math. 102(1), 49-56 (1992)

8. Grafakos, L, Torres, RH: On multilinear singular integrals of Calderón-Zygmund type. In: Proceedings of the 6th International Conference on Harmonic Analysis and Partial Differential Equations (El Escorual). Publ. Mat. Vol. Extra, 57-91 (2002)

9. Hedberg, LI: On certain convolution inequalities. Proc. Am. Math. Soc. 36, 505-510 (1972)

10. Hempel, J, Morris, G, Trudinger, N: On the sharpness of a limiting case of the Sobolev embedding theorem. Bull. Aust. Math. Soc. 3, 369-373 (1970)

11. Kenig, C, Stein, E: Multilinear estimates and fractional integration. Math. Res. Lett. 6, 1-15 (1999)

12. Lerner, AK, Ombrosi, S, Pérez, C, Torres, RH, Trujillo-González, R: New maximal functions and multiple weights for the multilinear Calderón-Zygmund theory. Adv. Math. 220(4), 1222-1264 (2009)

13. Moen, K: Weighted inequalities for multilinear fractional integral operators. Collect. Math. 60, 213-238 (2009)

14. Moser, J: A sharp form of an inequality by N. Trudinger. Indiana Univ. Math. J. 20, 1077-1092 (1971)

15. Muckenhoupt, B, Wheeden, R: Weighted norm inequalities for fractional integrals. Trans. Am. Math. Soc. 192, 261-274 (1974)

16. Li, W, Xue, Q, Yabuta, K: Multilinear Calderón-Zygmund operators on weighted Hardy spaces. Stud. Math. 199(1), 1-16 (2010)

17. $L u, G$, Yang, Y: Sharp constant and extremal function for the improved Moser-Trudinger inequality involving $L^{p}$ norm in two dimension. Discrete Contin. Dyn. Syst., Ser. A 25, 963-979 (2009)

18. Ruf, B: A sharp Trudinger-Moser type inequality for unbounded domains in $\mathbb{R}^{2}$. J. Funct. Anal. 219(2), 340-367 (2005)

19. Strichartz, RS: A note on Trudinger's extension of Sobolev's inequalities. Indiana Univ. Math. J. 21, $841-842$ (1972)

20. Tang, L: Endpoint estimates for multilinear fractional integrals. J. Aust. Math. Soc. 84, 419-429 (2008)

21. Tian, G, Zhu, X: A nonlinear inequality of Moser-Trudinger type. Calc. Var. Partial Differ. Equ. 10(4), $349-354$ (2000)

22. Trudinger, N: On imbedding into Orlicz spaces and some applications. J. Math. Mech. 17, 473-483 (1967)

doi:10.1186/1029-242X-2012-179

Cite this article as: Feng and Xue: Weighted Trudinger inequality associated with rough multilinear fractional type operators. Journal of Inequalities and Applications 2012 2012:179.

\section{Submit your manuscript to a SpringerOpen ${ }^{\odot}$ journal and benefit from:}

- Convenient online submission

- Rigorous peer review

Immediate publication on acceptance

- Open access: articles freely available online

- High visibility within the field

- Retaining the copyright to your article 